\title{
Análise da pluviosidade e do efeito de borda sobre os teores de flavonóides em Bauhinia cheilantha (Bong.) Steud., Fabaceae
}

\author{
Tadeu J. da S. Peixoto Sobrinho, ${ }^{1}$ Késsio de C. M. Cardoso, ${ }^{1}$ Tiago de L. B. Gomes, ${ }^{1}$ \\ Ulysses P. Albuquerque, ${ }^{2}$ Elba L. C. Amorim ${ }^{*}, 1$
}

\begin{abstract}
${ }^{1}$ Laboratório de Produtos Naturais, Departamento de Ciências Farmacêuticas, Universidade Federal de Pernambuco, Av. Prof. Arthur de Sá, s/n, Cidade Universitária, 50670-901 Recife-PE, Brasil ${ }^{2}$ Laboratório de Etnobotânica Aplicada, Departamento de Biologia, Universidade Federal Rural de Pernambuco, Av. Dom Manoel de Medeiros s/n, Dois Irmãos, 52171-900 Recife-PE, Brasil
\end{abstract}

\begin{abstract}
RESUMO: Este estudo avaliou a influência do efeito de borda e da pluviosidade sobre a produção de flavonóides em indivíduos de Bauhinia cheilantha (Bong.) Steud., Fabaceae, em uma área de caatinga no estado de Pernambuco, Nordeste do Brasil. Foi utilizada metodologia analítica por complexação com cloreto de alumínio para quantificar a concentração de flavonóides contidos nos extratos foliares de $B$. cheilantha, por meio de espectrofotometria no visível. De forma geral, o efeito de borda afeta a produção de flavonóides, entretanto, não foi possível correlacionar a produção de flavonóides e a pluviosidade, demonstrando que a espécie avaliada utiliza outra estratégia como resposta às pressões ambientais.
\end{abstract}

Unitermos: Caatinga, pata-de-vaca, pluviosidade, ecologia química, Bauhinia cheilantha, Fabaceae.

\begin{abstract}
Analysis of rainfall and edge-effects on flavonoid levels in Bauhinia cheilantha (Bong.) Steud., Fabaceae". The present study evaluated the influence of the edge-effect and rainfall on flavonoid content in individuals of Bauhinia cheilantha (Bong.) Steud., Fabaceae, in an area of caatinga vegetation in Pernambuco State, northeastern Brazil. The analytical methodology used aluminum chloride binding to quantify flavonoid concentrations by visible light spectrophotometry in leaf extracts of $B$. cheilantha. In general, forest edges influenced flavonoid production, but it was not possible to relate production with rainfall levels. These results demonstrate that this species uses various strategies to respond to environmental variables.
\end{abstract}

Keywords: Caatinga, pata-de-vaca, rainfall, chemical ecology, Bauhinia cheilantha, Fabaceae.

\section{INTRODUÇÃO}

Diversos fatores ambientais, como sazonalidade, ritmo circadiano, radiação, temperatura, altitude e umidade podem modificar o metabolismo secundário vegetal, interferindo quantitativamente e/ou qualitativamente na produção de compostos (Gobbo-Neto \& Lopes, 2007; Blank et al., 2007; Siqueira et al., 2007; Santos et al., 2009). Em situações de estresse (hídrico ou oxidativo, por exemplo) o vegetal pode modificar sua rota biossintética e produzir substâncias de defesa como respostas às mudanças de seu habitate (Dicke \& Hilker, 2003; Winkel-Shirley, 2002).

As variações sazonais como foto-período, intensidade luminosa e temperatura podem alterar significativamente os teores de vários grupos fenólicos, principalmente flavonóides, quando monitoradas em diferentes estações do ano (Yao et al., 2005). Por exemplo, diferenças significativas foram observadas no conteúdo de fenóis e flavonóides totais em Moringa oleifera Lam. (Moringaceae) em função da estação do ano e da condição climática em cinco regiões do Paquistão, onde áreas frias apresentaram maiores teores quando comparadas a regiões quentes, sendo estes efeitos de sazonalidade mais acentuados nos flavonóides (Iqbal \& Bhanger, 2006).

Duas espécies de samambaia, Pteridium caudatum (L.) Maxon e P. arachnoideum (Kaulf.) Maxon (Dennstaedtiaceae), foram o alvo de uma pesquisa sobre a influência da fenologia e a altitude em relação ao conteúdo de compostos fenólicos, percebendo-se um aumento progressivo com o desenvolvimento foliar em ambas (Alonso-Amelot et al., 2004). Contudo, há uma razão desigual entre a produção de compostos fenólicos de baixo peso molecular (BPM) e alto peso molecular (APM), verificando que a síntese e acumulação dos teores de compostos fenólicos de BPM independem da altitude, contrapondo os de APM que se correlacionaram 
positivamente com o aumento da altitude observados para as duas espécies (Alonso-Amelot et al., 2004).

Outro fator que interfere na síntese de metabólitos secundários é a fragmentação florestal, que por formar áreas de transição denominadas bordas, causam profundos impactos sobre os processos ecológicos, tornando-as vulneráveis à invasão de espécies exóticas e herbívoros, além de provocar alterações microclimáticas que exercem forte influência sobre o balanço energético da microrregião (Debinski \& Holt, 1999; Laurance \& Yensen, 1991; Murcia, 1995; Saunders et al., 1991). Deste modo, áreas fragmentadas com indivíduos localizados na borda e no interior de remanescentes florestais podem apresentar comportamento diferenciado em relação à produção de metabólitos secundários, como resposta às diferentes pressões ambientais. Estas mudanças, no entanto, não são permanentes e evoluem com o tempo à medida que a borda se fecha devido ao crescimento da vegetação (Périco et al., 2005).

No Brasil, há grande interesse em estudos relacionados à ecologia química, principalmente com vistas à identificação de aleloquímicos para a indústria de herbicidas e inseticidas (Silva et al., 2007; Baratto et al., 2008), entretanto, poucos estudos focalizam a ecologia química de plantas medicinais do bioma Caatinga. Monteiro et al. (2006) estudando os teores de taninos de Myracrodruon urundeuva (Engl.) Fr. All. (Anacardiaceae) e Anadenanthera colubrina (Vell.) Brenan (Mimosaceae) observaram que a síntese de substâncias tanantes possui forte relação com a sazonalidade e, estas espécies demonstram diferentes estratégias adaptativas frente a períodos de estiagem e chuva neste ecossistema.

Bauhinia cheilantha (Bongard) Steudel (Fabaceae) foi escolhida por ser uma espécie nativa da Caatinga, possuir forte pressão extrativista, ter elevada densidade na área de estudo (Alcoforado-Filho et al., 2003) e apresentar diversos usos na medicina tradicional (Albuquerque, 2006; Albuquerque et al., 2007a,b Agra et al., 2007a,b; Agra et al., 2008).

Um estudo dedicado a avaliar a relação entre os teores de flavonóides de $B$. cheilantha e fatores ambientais (efeito de borda e pluviosidade), fornecerá informações científicas essenciais para o entendimento da ecologia química de plantas da Caatinga, uma vez que poucos estudos dão ênfase a esta área e proporcionar às indústrias farmacêuticas o procedimento técnico para a realização eficiente do controle de qualidade das amostras, trazendo estabilidade, constância e eficácia aos produtos comercializados.

\section{MATERIAL E MÉTODOS}

\section{Área de estudo}

O trabalho foi desenvolvido no remanescente de Vegetação Caducifólia Espinhosa (Caatinga) com cerca de 20 ha pertencente à Estação Climatológica do Instituto Agronômico de Pernambuco (IPA), a nordeste de Caruaru e a aproximadamente $150 \mathrm{~km}$ de Recife, situada no Agreste pernambucano, conforme descrito em Monteiro et al., 2006. Segundo Alcoforado-Filho et al. (2003), este fragmento abriga cerca de 100 espécies representantes de árvores, arbustos, cipós e ervas distribuídas em 41 famílias, sendo os mais conspícuos, Euphorbiaceae, Mimosaceae e Fabaceae.

A área estudada possui solo podzólico amarelo, eutrófico, abrupto e textura franco-arenosa, a temperatura média anual varia entre $19-23{ }^{\circ} \mathrm{C}$, a precipitação média anual é abaixo de $700 \mathrm{~mm}$ e encontra-se a $537 \mathrm{~m}$ de altitude (08 14 '18,2'S e 35'54'57,1'W) (AlcoforadoFilho et al., 2003). A partir dos dados pluviométricos obtidos na Estação Climatológica do IPA, foi considerada estação seca o período entre os meses de outubro/2005 a março/2006 que apresentaram chuvas dispersas, enquanto que entre os meses de abril-setembro/2006 a precipitação total foi de $450,3 \mathrm{~mm}$.

\section{Coleta de material vegetal}

Para o monitoramento dos teores de flavonóides, foram formados dois grupos com dez indivíduos de $B$. cheilantha: Grupo I - formado por indivíduos selecionados dentro das parcelas previamente demarcadas, perfazendo área total de 1,0 ha (Araújo, 1998); Grupo II - formado por indivíduos selecionados na borda do fragmento, com distância máxima de $10 \mathrm{~m}$ da margem. Os critérios estabelecidos para coleta do material vegetal foram: folhas inteiras, localizadas nas extremidades dos ramos e mesmo estágio de desenvolvimento. As amostras coletadas foram armazenadas em sacos de papel, identificadas e levadas ao laboratório para subseqüente análise, sendo estas coletas realizadas entre os dias 18 e 22 de cada mês.

No mesmo período, as amostras coletadas foram identificadas pelo Prof. Dr. Ulysses P. de Albuquerque do Departamento de Biologia da Universidade Federal Rural de Pernambuco e as exsicatas encontram-se incorporadas ao Herbário Prof. Vasconcelos Sobrinho do Departamento de Botânica, da Universidade Federal Rural de Pernambuco sob os no. 49684-49704.

\section{Procedimentos experimentais}

\section{Preparação dos extratos}

As amostras vegetais coletadas em campo foram transportadas ao laboratório, no qual passaram pelo processo de secagem em estufa durante três dias a $50{ }^{\circ} \mathrm{C}$, pulverizadas em triturador industrial e analisadas quantitativamente. Os extratos foram preparados em erlenmeyers contendo $500 \mathrm{mg}$ das amostras pulverizadas e $25 \mathrm{~mL}$ de metanol em placa de aquecimento a $80 \pm 5^{\circ} \mathrm{C}$ durante $30 \mathrm{~min}$. Os extratos foram filtrados em papel de 
filtro quantitativo e transferidos para balões volumétricos de $50 \mathrm{~mL}$. O resíduo do material foi lavado com $25 \mathrm{~mL}$ de metanol, filtrado para o mesmo balão e completado o volume com metanol. Todos os procedimentos foram realizados em triplicata.

\section{Quantificação do conteúdo flavônico}

Seguiu-se a metodologia descrita por Peixoto Sobrinho et al. (2008). Do extrato preparado foram pipetadas e transferidas alíquotas de $1,0 \mathrm{~mL}$ para balões volumétricos de $25 \mathrm{~mL}$, ao qual foram adicionados 0,6 $\mathrm{mL}$ de ácido acético glacial, $10 \mathrm{~mL}$ do reagente piridina (solução metanólica a $20 \% \mathrm{v} / \mathrm{v}$ ) e $2,5 \mathrm{~mL}$ do reagente cloreto de alumínio (solução metanólica a $50 \mathrm{mg} / \mathrm{L} \mathrm{p} / \mathrm{v}$ ), completando-se o volume do balão com água destilada. Após intervalo de $30 \mathrm{~min}$ a temperatura ambiente e ao abrigo da luz, as leituras foram realizadas a $420 \mathrm{~nm}$ com cubeta de quartzo de $10 \mathrm{~mm}$ de caminho óptico. Utilizouse solução metanólica de Rutina como padrão $(0,5 \mathrm{mg} /$ $\mathrm{mL}$ ) para construir a curva de calibração, utilizando-se seis concentrações: 6,$0 ; 8,0 ; 10,0 ; 12,0 ; 16,0 ; 20,0 \mu \mathrm{g} / \mathrm{mL}$. Os resultados foram expressos em teor $(\mathrm{p} / \mathrm{p})$ de matéria seca.

\section{Análise estatística}

Para avaliar a normalidade dos dados foi empregado o teste Kolmogorov-Smirnov (Ayres et al., 2005). A análise de variância ANOVA um critério foi utilizada para comparar, individualmente, as diferenças nos teores de flavonóides intra-grupo (Grupo I e Grupo II) durante os meses de coleta e para confrontar os resultados inter-grupo (Grupo I versus Grupo II), complementandose o estudo, quando necessário, com o teste de Tukey para comparar as diferenças entre as médias. Teste de correlação foi usado para avaliar se os teores de flavonóides dos Grupos I (interior) e II (borda) relacionavam-se entre si e, se estes foram influenciados pela pluviosidade. Todas as análises foram realizadas utilizando-se o programa BioEstat 4.0 (Ayres et al., 2005).

\section{RESULTADOS}

O teste Kolmogorov-Smirnov não encontrou valores unilaterais e bilaterais significativos, indicando que os dados possuem distribuição normal.

Foi observado que durante o monitoramento mensal, os teores de flavonóides de $B$. cheilantha variaram intra-grupo, sendo observadas diferenças estatísticas no Grupo I $(\mathrm{p}<0,0001)$ e no Grupo II $(\mathrm{p}<0,0001)$. Através da análise de variância realizada entre os Grupos I e II, pode-se confirmar que os indivíduos localizados à borda do fragmento concentraram mais flavonóides do que os do interior da mata $(\mathrm{p}<0,0001)$. Porém, este padrão não ocorreu no mês de julho/2006, quando o teor de flavonóides do Grupo I foi mais elevado. O coeficiente de correlação de Pearson apontou relação positiva entre os grupos $(\mathrm{r}=0,8391 ; \mathrm{p}<0,05)$.

Os teores de flavonóides foliares de $B$. cheilantha do Grupo I correlacionaram-se negativamente com a precipitação acumulada de 30 dias $(r=-0,2589 ; \mathrm{p}=0,620)$, o que também foi observado com o Grupo II ( $\mathrm{r}=-0,1605$; $\mathrm{p}=0,761)$. Contudo, não ficou evidente a relação existente entre a pluviosidade e a produção de flavonóides, pois os resultados não apresentaram estatísticas significativas. Os dados podem ser visualizados na Tabela 1 e na Figura 1.

Tabela 1. Média mensal seguida de erro-padrão dos níveis de flavonóides de dez indivíduos de Bauhinia cheilantha (Bong.) Steud. coletados no interior (Grupo I) e borda (Grupo II) na área de estudo em Caruaru-PE, Brasil.

\begin{tabular}{lcc}
\hline \multirow{2}{*}{ Mês } & \multicolumn{2}{c}{ Teor de Flavonóides $(\% \mathrm{p} / \mathrm{p})$} \\
\cline { 2 - 3 } & Grupo I $(\bar{\chi} \pm \mathrm{EP})$ & Grupo I $(\bar{\chi} \pm \mathrm{EP})$ \\
\hline Abril & $2,117 \pm 0,301 \mathrm{ac}$ & $2,213 \pm 0,396 \mathrm{ac}$ \\
Maio & $1,641 \pm 0,835 \mathrm{a}$ & $2,291 \pm 1,018 \mathrm{ac}$ \\
Junho & $3,334 \pm 0,505 \mathrm{~b}$ & $3,557 \pm 0,856 \mathrm{~b}$ \\
Julho & $2,244 \pm 0,392 \mathrm{ac}$ & $1,839 \pm 1,077 \mathrm{c}$ \\
Agosto & $2,974 \pm 0,292 \mathrm{bd}$ & $3,640 \pm 0,412 \mathrm{~b}$ \\
Setembro & $2,479 \pm 0,280 \mathrm{~cd}$ & $2,991 \pm 0,425 \mathrm{ab}$ \\
\hline
\end{tabular}

Médias seguidas da mesma letra na coluna não diferem estatisticamente entre si $(\mathrm{p}<0,05)$.

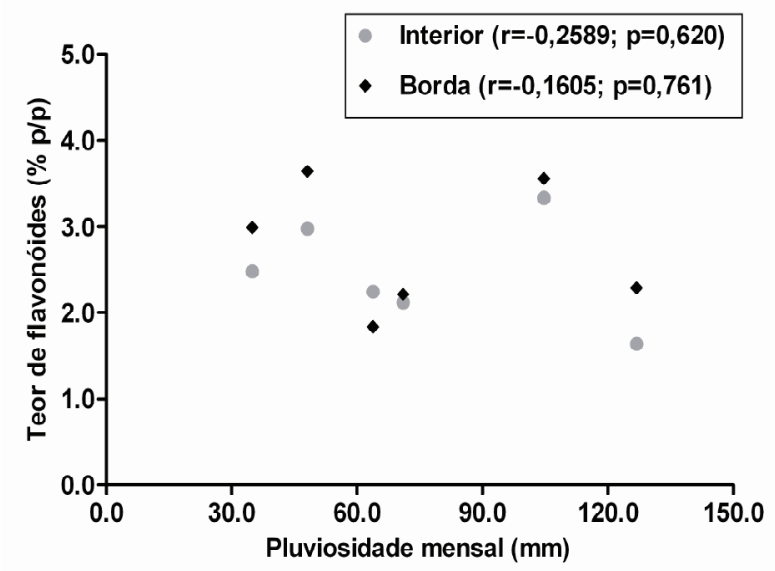

Figura 1. Correlação entre teor de flavonóides foliares (\% p/p) de Bauhinia cheilantha (Bong.) Steud. em relação à pluviosidade num fragmento de mata de Caatinga no município de CaruaruPE, região Nordeste do Brasil.

\section{DISCUSSÃO}

O efeito de borda (Debinski \& Holt, 2000; Laurance \& Yensen, 1991; Murcia, 1995; Saunders et al., 1991) parece explicar o comportamento apresentado por indivíduos de $B$. cheilantha localizados na borda do fragmento (Grupo II) em comparação com os do interior (Grupo I), em função da produção de flavonóides, onde 
os indivíduos do grupo II exibiram concentrações mais elevadas destes compostos. No entanto, esta influência não foi observada no mês de julho/2006, quando o Grupo I apresentou teores mais elevados de compostos flavônicos.

Embora este fenômeno possa explicar os resultados encontrados, fatores intrínsecos e extrínsecos podem interferir na produção de flavonóides. Por exemplo, indivíduos localizados na borda recebem maior incidência luminosa causando modificações fisiológicas nas folhas, o que pode atrair insetos herbívoros e estes, atrair pássaros (Barbosa et al., 2005; Murcia, 1995). Assim, o efeito de borda em função da radiação e ataque de herbívoros, pode desencadear uma série de respostas quantitativas e/ ou qualitativas (Dicke \& Hilker, 2003), como a produção de flavonóides, que fornecem proteção a herbivoria e radiação ultravioleta (Amaral et al., 2004), podendo ser estes os fatores que influenciam a síntese deste grupo em indivíduos de B. cheilantha.

Foi observado que o processo de herbivoria promove diversificação na produção de substâncias de defesa, como flavonas, flavonóis e flavanonas em folhas atacadas de Lonchocarpus muehlbergianus Hassl (Fabaceae), sugerindo que o vegetal produz defesas mais eficientes ao ataque do herbívoro (Oliveira et al., 2006). Confirmando estes resultados, Soares et al. (2000), verificaram que folhas sadias e folhas atacadas de Rollinia laurifolia Schdtl. (Annonaceae), apresentavam perfis flavônicos distintos, mostrando que as amostras herbivoradas possuíam maior diferenciação estrutural, indicando resposta qualitativa.

A produção de flavonóides de $B$. cheilantha não parece estar relacionada com a pluviosidade, embora para outras espécies vegetais seja observada esta relação. Por exemplo, para amostras de Cecropia glaziovii Snethl. (Cecropiaceae) coletadas em três regiões de Minas Gerais/ Brasil os teores de flavonóides em folhas jovens e maduras foram mais elevados na estiagem quando comparados com o período chuvoso (Luengas-Caicedo et al., 2007). Estudo realizado com Baccharis trimera Less. (Asteraceae) mostrou que os teores de flavonóides das amostras coletadas no verão diferiram estatisticamente das demais, representando aumento médio de $72 \%$ das amostras coletadas no inverno e $80 \%$ da primavera (Borella et al., 2001).

Solar et al. (2006) observaram que ao longo das estações pode haver diferenças no teor de substâncias fenólicas em Juglans regia L. (Juglandaceae), verificando um aumento progressivo no conteúdo de flavonóides com o início da estiagem, enquanto que para alguns fenóis (vanílico, siríngico, elágico e clorogênico) foi o oposto, decrescendo os teores de flavonóides com a estiagem.

Sosa et al. (2005) estudando dois grupos de Cecropia glaziovii Snethl. (Cecropiaceae) provenientes de duas regiões da Espanha, com condições climáticas diferentes, observaram que em folhas maduras, as quantidades de flavonóides quadruplicaram entre o inverno e o verão no Grupo I (menor pluviosidade), enquanto que no Grupo II (maior pluviosidade) esse aumento foi três vezes maior, com diferenças significativas entre os grupos. Neste mesmo estudo, foi observado que para as folhas senescentes, os teores de flavonóides foram superiores aos encontrados nas folhas maduras, em relação à massa do exudato, do outono a primavera (Sosa et al., 2005).

A sazonalidade climática parece ser um importante fator também para taninos, como foi verificado por Monteiro et al. (2006) com duas espécies típicas do bioma Caantiga. Os autores observaram que cascas e folhas de Anadenanthera colubrina (Vell.) Brenan (Mimosaceae) e Myracrodruon urundeuva (Engl.) Fr. All. (Anacardiaceae), concentravam mais taninos nos períodos de estiagem, verificando também que as cascas de $A$. colubrina possuíam maiores teores do que as folhas durante a estação chuvosa, enquanto que $M$. urundeuva exibiu teores mais elevados nas suas folhas, em comparação com suas cascas, durante a estação chuvosa (Monteiro et al., 2006).

Este trabalho gerou contribuições essenciais para a compreensão da ecologia química de plantas da Caatinga, uma vez que há poucas pesquisas relacionadas à importância deste ecossistema sobre a produção de metabólitos secundário, em especial flavonóides. As diferenças nos teores de flavonóides observadas, entre indivíduos localizados no interior e borda da mata, podem fornecer parâmetros para a busca com mais segurança da matéria-prima vegetal, melhorando a qualidade dos produtos comercializados e diminuindo a pressão extrativista.

\section{AGRADECIMENTOS}

Os autores agradecem ao apoio financeiro do Conselho Nacional de Desenvolvimento Científico e Tecnológico (CNPq) pelo auxílio financeiro, a Coordenação de Aperfeiçoamento de Pessoal de Nível Superior (CAPES) pela concessão da bolsa ao primeiro autor, ao Instituto Agronômico de Pernambuco (IPA) e aos funcionários da Estação Climatológica de Caruaru pelo apoio logístico.

\section{REFERÊNCIAS}

Agra MF, Freitas PF, Barbosa Filho JM 2007a. Synopsis of the plants known as medicinal and poisonous in Northeast of Brazil. Rev Bras Farmacogn 17: 114-140.

Agra MF, Baracho GS, Nurit K, Basílio IJLD, Coelho VPM 2007b. Medicinal and poisonous diversity of the flora of "Cariri Paraibano", Brazil. J Ethnopharmacol 111: 383395.

Agra MF, Silva KN, Basílio IJLD, França PF, Barbosa-Filho JM 2008. Survey of medicinal plants used in the region Northeast of Brazil. Rev Bras Farmacogn 18: 472-508.

Albuquerque UP 2006. Re-examining hypotheses concerning the use and knowledge of medicinal plants: a study in the Caatinga vegetation of NE Brazil. J Ethnobiol Ethnomed 


$$
\text { 2: } 1-10 \text {. }
$$

Albuquerque UP, Monteiro JM, Ramos MA, Amorim ELC 2007a. Medicinal and magic plants from a public market in northeastern Brazil. J Ethnopharmacol 110: 76-91.

Albuquerque UP, Medeiros PM, Almeida ALS, Monteiro JM, Lins Neto EMF, Melo JG, Santos JP 2007b. Medicinal plants of the Caatinga (semi-arid) vegetation of $\mathrm{NE}$ Brazil: a quantitative approach. J Ethnopharmacol 114: 325-354.

Alcoforado-Filho FG, Sampaio EVSB, Rodal MJN 2003 Florística e fitossociologia de um remanescente de vegetação caducifólia espinhosa arbórea em Caruaru, Pernambuco. Acta Bot Bras 17: 287-303.

Alonso-Amelot ME, Oliveros A, Calcagno-Pisarelli MP 2004 Phenolics and condensed tannins in relation to altitude in neotropical Pteridium spp. A field study in the Venezuelan Andes. Biochem Syst Ecol 32: 969-981.

Amaral JS, Seabra RM, Andrade PB, Valentão P, Pereira JA, Ferreres, F 2004. Phenolic profile in the quality control of walnut (Juglans regia L.) leaves. Food Chem 88: 373379.

Araújo EL 1998. Aspectos da dinâmica populacional de duas espécies em floresta tropical seca (caatinga), Nordeste do Brasil. Tese de Doutorado - Universidade Estadual de Campinas.

Ayres M, Ayres MJ, Ayres DL, Santos SA 2005. BioEstat 4.0: aplicações estatísticas nas áreas das ciências biomédicas. Belém: Sociedade Civil/Instituto de Desenvolvimento Sustentável Mamirauá.

Baratto L, Lang KL, Vanz DC, Reginatto FH, Oliveira JB, Falkenberg M 2008. Investigação das atividades alelopática e antimicrobiana de Mikania laevigata (Asteraceae) obtida de cultivos hidropônico e tradicional. Rev Bras Farmacogn 18: 577-582.

Barbosa VLS, Leal IR, Iannuzzi L, Almeida-Cortez J 2005. Distribution pattern of herbivorous insects in a remnant of Brazilian Atlantic forest. Neotrop Entomol 34: 701711.

Blank AF, Costa AG, Arrigoni-Blank MF, Cavalcanti SCH, Alves PB, Innecco R, Ehlert PAD, Sousa IF 2007. Influence of season, harvest time and drying on Java citronella (Cymbopogon winterianus Jowitt) volatile oil. Rev Bras Farmacogn 17: 557-564.

Borella JC, Fontoura A, Menezes Jr A, França SC 2001. Influência da adubação mineral (N-P-K) e sazonalidade no rendimento e teor de flavonóides em indivíduos masculinos de Baccharis trimera (Asteraceae) - Carqueja. Rev Bras Plant Med 4: 99-102.

Debinski DM, Holt RD 2000. A survey and overview of habitat fragmentation experiments. Conserv Biol 14: 342-355.

Dicke M, Hilker M 2003. Induced plant defences: from molecular biology to evolutionary ecology. Basic Appl Ecol 4: 3-14.

Gobbo-Neto L, Lopes NP 2007. Plantas medicinais: fatores de influência no conteúdo de metabólitos secundários. Quim Nova 30: 374-381.
Iqbal S, Bhanger MI 2006. Effect of season and production location on antioxidant activity of Moringa oleifera leaves grown in Pakistan. J Food Comp Anal 19: 544551.

Laurance WF, Yensen E 1991. Predicting the impacts of edge effects in fragmented habitats. Biol Conserv 55: 77-92.

Luengas-Caicedoa PE, Braga FC, Brandão GC, Oliveira AB 2007. Seasonal and intraspecific variation of flavonoids and proanthocyanidins in Cecropia glaziovi Sneth. leaves from native and cultivated specimens. Z Naturforsch $62 c$ : 701-709.

Monteiro JM, Albuquerque UP, Neto EMFL, Araújo EL, Albuquerque MM, Amorim ELC 2006. The effects of seasonal climate changes in the Caatinga on tannin levels in Myracrodruon urundeuva (Engl.) Fr. All. and Anadenanthera colubrina (Vell.) Brenan. Rev Bras Farmacogn 16: 338-344.

Murcia C 1995. Edge effects in fragmented forests: implications for conservation. Tree 10: 58-62.

Oliveira DC, Christiano JCS, Soares GLG, Isaias RMS 2006. Reações de defesas químicas e estruturais de Lonchocarpus muehlbergianus Hassl. (Fabaceae) à ação do galhador Euphalerus ostreoides Crawf. (Hemiptera: Psyllidae). Rev Bras Bot 29: 657-667.

Peixoto Sobrinho TJS, Silva CHTP, Nascimento JE, Monteiro JM, Albuquerque UP, Amorim, ELC 2008. Validação de metodologia espectrofotométrica para quantificação dos flavonóides de Bauhinia cheilantha (Bongard) Steudel. Rev Bras Cienc Farm 44: 683-689.

Périco E, Cemin G, Lima DFB, Rempel C 2005. Efeitos da fragmentação de hábitats sobre comunidades animais: utilização de sistemas de informação geográfica e de métricas de paisagem para seleção de áreas adequadas a testes. Anais do XII Simpósio Brasileiro de Sensoriamento Remoto, Goiânia INPE: 2339-2346.

Santos A, Paduan RH, Gazin ZC, Jacomassi E, D' Oliveira PS, Cortez DAG, Cortez LER 2009. Determinação do rendimento e atividade antimicrobiana do óleo essencial de Cymbopogon citratus (DC.) Stapf em função de sazonalidade e consorciamento. Rev Bras Farmacogn 19: 436-441.

Saunders DA, Hobbs RJ, Margules CR 1991. Biological consequences of ecosystem fragmentation: a review. Conserv Biol 5: 18-32.

Silva JP, Crotti AEM, Cunha WR 2007. Antifeedant and allelopathic activities of the hydroalcoholic extract obtained from Neem (Azadirachta indica) leaves. Rev Bras Farmacogn 17: 529-532.

Siqueira EP, Alves TMA, Zani CL 2007. Fingerprint of volatiles from plant extracts based on SPME-GC-MS. Rev Bras Farmacogn 17: 565-571.

Soares GLG, Isaias RMS, Gonçalves SJMR, Christiano JCS 2000. Alterações químicas induzidas por coccídeos galhadores (Coccoidea, Brachyscelidae) em folhas de Rollinia laurifolia Schdtl. (Annonaceae). Rev Bras Zoociências 2: 103-133. 
Solar A, Colaric M, Usenik V, Stampar F 2006. Seasonal variations of selected flavonoids, phenolic acids and quinines in annual shoots of common walnut (Juglans regia L.). Plant Sci 170: 453-461.

Sosa T, Alías JC, Escudero JC, Chaves N 2005. Interpopulational variation in the flavonoid composition of Cistus ladanifer L. exudate. Biochem Syst Ecol 33: 353-364.

Winkel-Shirley B 2002. Biosynthesis of flavonoids and effects of stress. Plant Biol 5: 218-223.

Yao L, Caffin N, D’Arcy B, Jiang Y, Shi J, Singanusong R, Liu X, Datta N, Kakuda Y, Xu Y 2005. Seasonal variations of phenolic compounds in Australia-grown tea (Camellia sinensis). J Agric Food Chem 53: 6477-6483. 